\title{
Joint effect of maternal plasma homocysteine and prepregnancy obesity on child blood pressure: A prospective birth cohort study
}

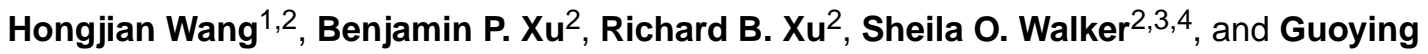 \\ Wang ${ }^{2, *}$ \\ ${ }^{1}$ Department of Cardiovascular Internal Medicine, State Key Laboratory of Cardiovascular \\ Disease, National Clinical Research Center of Cardiovascular Diseases, Fuwai Hospital, National \\ Center for Cardiovascular Diseases, Chinese Academy of Medical Sciences and Peking Union \\ Medical College, Beijing, China \\ ${ }^{2}$ Department of Population, Family and Reproductive Health, Johns Hopkins University \\ Bloomberg School of Public Health, Baltimore, MD, USA \\ ${ }^{3}$ School of Education, Johns Hopkins University, Baltimore, MD, USA \\ ${ }^{4}$ University of California, Irvine, Irvine, USA
}

\begin{abstract}
Background/Objectives-Elevated homocysteine (Hcy) is a known cardiovascular risk factor. However, its role in intergenerational cardiometabolic risk is unknown. We hypothesized that maternal elevated Hcy can act alone or in combination with maternal prepregnancy obesity to increase child systolic blood pressure (SBP).
\end{abstract}

Methods-This study included 1279 mother-child pairs who were enrolled at birth and followed prospectively up to age 9 years from 2003-2014 at the Boston Medical Center. Child SBP percentile was calculated according to U.S. reference data and elevated SBP was defined as SBP $\geq 75^{\text {th }}$ percentile.

Results-A U-shaped relationship between maternal Hcy and her child SBP was observed. The risk for child elevated SBP was higher amongst those in the lowest quartile (Q1, odds ratio $(\mathrm{OR})$ : 1.27; 95\% confidence interval (CI): 0.94 - 1.72), and highest quartile (Q4, OR: 1.34; 95\% CI: 1.00 -1.81) as compared with those in quartiles 2 and 3. The highest risk of child elevated SBP was found amongst children born to obese mothers with Hcy in Q4 (OR: 2.22; 95\%CI: 1.35 - 3.64), compared with children of non-obese mothers with Hcy in Q2-Q3. This association was

\footnotetext{
Users may view, print, copy, and download text and data-mine the content in such documents, for the purposes of academic research, subject always to the full Conditions of use: http://www.nature.com/authors/editorial_policies/license.html\#terms

*Correspondence and reprint requests should be addressed to: Guoying Wang, MD, PhD, Department of Population, Family and Reproductive Health, Johns Hopkins University Bloomberg School of Public Health, 615 N. Wolfe Street, Baltimore, MD 21205-2179, Phone: 410-502-8973, Fax: 410-502-5831, gwang24@jhu.edu.

Conflict of interest: The authors declare no conflict of interest.

Supplementary information: Supplemental Figure and Tables; MS Word documents (.docx); Supplementary information is available at International Journal of Obesity's website
} 
independent from maternal folate and vitamin B12 status, and was not mediated by gestational age or size at birth.

Conclusion-In this prospective birth cohort, we observed a U- shaped association between maternal Hcy levels and child elevated SBP. Maternal high Hcy (Q4) and prepregnancy obesity jointly increased the risk of child elevated SBP by more than two fold.

\section{Keywords}

maternal homocysteine; prepregnancy obesity; child systolic blood pressure

\section{Introduction}

Homocysteine (Hcy), which by itself can cause endothelial cell injury, ${ }^{1-3}$ is an intermediate molecule of methionine metabolism and is elevated in the presence of folate and/or vitamin B12 deficiencies. ${ }^{4}$ Elevated Hcy is a known cardiovascular risk factor. For example, total Hcy is a well-established marker of increased stroke risk via its role in accelerating atherosclerotic disease. ${ }^{5,6}$ Some studies have identified a direct relationship between plasma Hcy concentrations, systolic blood pressure and hypertension. ${ }^{7,}{ }^{8}$ In the third National Health and Nutrition Examination Survey, the prevalence of hypertension was a 2- to 3-fold higher among individuals in the highest quintile of plasma Hcy compared to those in the lowest quintile. ${ }^{9}$ However, the role of Hcy in intergenerational cardiometabolic risk is unknown. Several studies showed that the risk of pregnancy complications and adverse pregnancy outcomes including low birthweight and intrauterine growth restriction were increased among women with elevated Hcy concentration in the third trimester. ${ }^{10,11}$ Furthermore, these complications and outcomes were associated with cardiovascular disease in children. We speculated that maternal Hcy concentration during late pregnancy may have a long-term influence on child cardiometabolic health - including key risk factors such as blood pressure.

Even in normal pregnancy, the blood levels of folate, cobalamin, and pyridoxal 5-phosphate tend to go down, ${ }^{12}$ leading to a high total oxidation status. In the presence of insufficient intake of these essential micronutrients, maternal oxidative stress may further increase, which may impair fetal growth, potentially constituting a developmental risk factor for adult disease. In addition, oxidative stress increases DNA damage. ${ }^{13}$ In a pilot randomized controlled trial of pregnant women ${ }^{14}$, continued supplementation with $400 \mu \mathrm{g}$ folate/d in the $2^{\text {nd }}$ and $3^{\text {rd }}$ trimesters increased maternal and cord blood folate status, and prevented the increase in Hcy concentration in late pregnancy. However, there is little clinical and epidemiological evidence about whether maternal Hcy concentrations during pregnancy particularly in the $3^{\text {rd }}$ trimester - can influence childhood health outcomes. Moreover, it remains to be determined whether an elevated Hcy concentration is only a reflection of folate status, or whether it has an independent effects on the vascular system.

In the U.S., obesity affects over one third of the women of childbearing age. ${ }^{15}$ Maternal prepregnancy obesity has been linked to higher offspring systolic blood pressure (SBP), likely through intrauterine mechanisms. ${ }^{16,17}$ In animal and human studies, maternal obesity also has been associated with high blood Hcy levels. ${ }^{18,19}$ From this perspective, studying 
the association between maternal Hcy and child BP in the presence and absence of maternal prepregnancy obesity could provide new insight into their joint association with child BP.

Using a large, existing U.S. prospective birth cohort, the current study sought to test the hypotheses that maternal elevated Hcy can act independently and/or jointly with maternal prepregnancy obesity to increase child SBP at age 3-9 years.

\section{Methods}

\section{Study Participants}

The Boston Birth Cohort (BBC) was initiated in 1998 with a rolling enrollment protocol. The BBC's largely urban, low-income minority population has been described previously; ${ }^{20}$ the current study included mother-infant pairs from this cohort. At enrollment, within 48 -72 hours of delivery, mothers responded to a standardized questionnaire to assess maternal demographic and environmental information, including prepregnancy weight, height, race/ ethnicity, education, smoking status, alcohol intake, parity, perceived stress during pregnancy, and prenatal multivitamin intake. The research team also drew blood from all mothers at enrollment. Since 2003, children were followed if they were enrolled in the BBC and planned to receive primary care at Boston Medical Center (BMC). Information on postnatal demographics and environment was also collected using a standardized questionnaire. The study protocol was approved by the Institutional Review Boards of Boston University Medical Center, Ann \& Robert H. Lurie Children's Hospital of Chicago, and the Johns Hopkins Bloomberg School of Public Health. All of the study mothers provided written informed consent.

Figure S1 displays how participants were selected for this analysis. Of the 7939 motherchild pairs enrolled in the BBC, 1877 were followed from 2003 to 2014 and completed at least one postnatal well-child visit with BP measurement between ages 3 and 9 at BMC. The current analysis was further restricted to 1279 mother-child pairs who had complete data on prepregnancy BMI, diabetes (either pre-existing or gestational diabetes), hypertensive disorders in pregnancy, gestational age, birthweight, and maternal plasma Hcy. The demographic characteristics and birth outcomes of the mothers were comparable between participants who were included and excluded from the study (Table S1).

\section{Maternal Variables}

A standard maternal questionnaire interview was used to determine maternal prepregnancy weight and height within 2-3 days of delivery. Maternal body mass index (BMI) was calculated as weight in kilograms divided by height in meters squared $\left(\mathrm{kg} / \mathrm{m}^{2}\right)$, and then categorized into three groups: normal weight $\left(18.5-24.9 \mathrm{~kg} / \mathrm{m}^{2}\right)$, overweight $(25-29.9 \mathrm{~kg} /$ $\mathrm{m}^{2}$ ), and obesity ( $>30 \mathrm{~kg} / \mathrm{m}^{2}$ ) using criteria from the Centers for Disease Control and Prevention. ${ }^{21}$ Because the effects of normal weight and overweight on child SBP were similar (data not shown), prepregnancy BMI was re-categorized into two groups: non-obese $\left(18.5-29.9 \mathrm{~kg} / \mathrm{m}^{2}\right)$ and obese $\left(\geq 30 \mathrm{~kg} / \mathrm{m}^{2}\right)$ in the subsequent analyses. Underweight mothers were not included in the analysis due to a small sample size $(n=44)$. 
Maternal race/ethnicity was classified as Black, Hispanic, or Other (which included White, Asian, Pacific Islander, and mothers who reported more than one race). Maternal education level was categorized into high school and below vs. college and above. The information on maternal smoking and alcohol intake was based on maternal self-report during a standardized questionnaire interview within a few days of delivery. Maternal smoking during pregnancy was classified into three groups: never smoker (did not smoke cigarettes at any time during the index pregnancy), quitter (only smoked in the three months leading up to the pregnancy or during the $1^{\text {st }}$ trimester), or continuous smoker (smoked from prepregnancy to delivery). ${ }^{22}$ We defined alcohol intake as "No" for those women who did not drink or only occasionally drank alcohol during their entire pregnancy. We defined intake as "Yes" for those who regularly drank alcohol during pregnancy. Perceived stress during pregnancy was categorized into low vs. high. ${ }^{23}$ Maternal hypertensive disorders of pregnancy ${ }^{24}$ included one or more of the following: pregnancy-induced hypertension (i.e., gestational hypertension, preeclampsia, eclampsia and HELLP syndrome) as well as hypertension that existed prior to pregnancy (referred to here as existing hypertension). Maternal diabetes status was classified as nondiabetic or diabetic (either pre-existing or gestational diabetes). ${ }^{20}$

Gestational age was determined based on the first day of the last menstrual cycle and by early prenatal ultrasonographic results. ${ }^{22}$ Infants were then categorized into term ( $237 \mathrm{wks}$ ) and preterm $(<37 \mathrm{wks}) .{ }^{20}$ Birthweight was abstracted from the electronic medical record (EMR). Birthweight for gestational age was classified into three groups: small for gestational age (SGA; <10th percentile), large for gestational age (LGA; >90th percentile), and appropriate for gestational age (AGA; 10th to 90th percentile) according to an established local gender- and race-specific reference population. ${ }^{25}$

\section{Anthropometric outcomes and blood pressure}

Child weight, height and BP were measured by medical staff during well-child visits as documented in the EMR. Height and weight $z$-scores were calculated using U.S. reference data. ${ }^{26}$ For our primary outcome variable, we focused on childhood systolic BP rather than diastolic BP because it is a better predictor of later outcomes and is more accurately measured. ${ }^{27,} 28$ In the BMC, child SBP was measured using an appropriate sized cuff, and measured at the right brachial artery using the validated automatic sphygmomanometer Masimo Set (2003-2008: the Welch Allyn 420 Spot Vital Signs monitor; 2008-2014, the Welch Allyn 45MT0 Spot Vital Signs LXi monitor). SBP percentile was calculated using a U.S. national reference for age, gender and height. ${ }^{29} \mathrm{In}$ accordance with earlier definitions used amongst pediatric populations to define childhood metabolic syndrome-like phenotype ${ }^{30}$ we defined elevated SBP as SBP $\geq 75$ th percentile.

\section{Ascertainment of plasma Hcy, folate and vitamin B12 concentrations}

Plasma Hcy was measured using automatic clinical analyzers (BeckmanCoulter) at the core laboratory of the National Clinical Research Center for Kidney Disease, Nanfang Hospital, Guangzhou, China. ${ }^{31}$ Folate and vitamin B12 were measured by a commercial laboratory using chemiluminescent immunoassay on a MAGLUMI 2000 Analyzer (Snibe Co., Ltd) with an inter-assay coefficient of variation of less than $4 \% .32$ 


\section{Statistical Analysis}

Our primary outcome of interest was SBP measured at the last well-child visit. We modeled SBP percentile (continuous variable) and elevated SBP (binary variable). The primary predictor that we assessed was maternal Hcy concentration.

As a first step, we examined the linear association between maternal plasma Hcy and child SBP percentile using smoothing plots (PROC LOESS). Due to the U-shaped relationship between Hcy and SBP, we analyzed Hcy as a categorical variable. We modeled Hcy in quartiles (Qs) and grouped them into three categories for all of the regression analyses: low (Q1: 3.14-6.27umol/L), adequate (Q2-Q3:6.28-9.57umol/L) and high

(Q4:9.58-22.59umol/L). We use Q2-Q3 as the reference category in the analyses, and this was used to address the question "Are low or high maternal Hcy levels associated with higher child SBP?"

Next, we estimated the individual and joint association between maternal Hcy and maternal prepregnancy obesity on child SBP using linear regression models, and child elevated SBP using logistic regression models. We assessed the interaction effect of maternal prepregnancy obesity $(0=\mathrm{No}, 1=\mathrm{Yes})$ and Hcy status on child elevated SBP percentiles and assessed the odds of elevated SBP by including two cross-product terms in the models, with indicator terms for maternal prepregnancy obesity and Hcy status.

In the final step, we assessed the independent effect of maternal Hcy on child SBP with additional adjustment for: 1) maternal plasma folate and vitamin B12 levels during pregnancy; and 2) birth outcome (preterm and body size at birth). To further evaluate the strength of the findings, we conducted stratified analyses by race (Black only, the majority race group in the BBC) and child age group (ages 6-9 only).

For all analyses using regression models, we adjusted for maternal age, race, education, alcohol intake, smoking, parity, perceived stress during pregnancy, diabetes and hypertensive disorders. Covariates were selected based on previous literature documenting their association with exposures and outcomes of interest in our study. We additionally adjusted for child plasma Hcy, and investigated the combined effect of child Hcy and maternal Hcy on child SBP percentile and the odds of elevated SBP. Child age, sex and height were excluded from the regression models because they had already been accounted for when we defined the outcome variables. All of the statistical tests were two-sided and significance was set at $\mathrm{p}<0.05$. All statistical analyses were performed using SAS (SAS Institute), version 9.4 .

\section{Results}

This study was comprised of 1279 mother-child pairs, including 864 Black (67.6\%) and 247 Hispanic (19.3\%). In all, 322 (25.2\%) mothers were obese in prepregnancy, and a total of 369 (28.9\%) children had elevated SBP at ages 3 to 9. Children with elevated SBP had a lower birthweight, a shorter gestational age, and a higher current BMI. They were also more likely to have mothers with prepregnancy obesity and hypertensive disorders (Table 1). The 
median (interquartile range [IQR]) for maternal plasma Hcy concentrations was $7.55 \mathrm{umol} / \mathrm{L}$ (IQR: 6.28-9.57 umol/L).

\section{Maternal homocysteine concentration, maternal prepregnancy obesity and child SBP}

A U-shaped association between maternal Hcy concentration and child SBP percentile was observed. Figure 1 shows the association between maternal Hcy and child SBP percentile stratified by prepregnancy BMI category in this cohort. The lowest child SBP levels appeared in children whose mothers had Hcy levels in Q2-Q3 (6.28-9.57 umol/L), and higher child SBP levels were seen in mothers with low $(\mathrm{Q} 1: 3.14-6.27 \mathrm{umol} / \mathrm{L})$ and high $(\mathrm{Q} 4$ : 9.58-22.59umol/L) Hcy concentrations, especially in mothers with prepregnancy obesity. Because similar patterns were observed in both genders, the following analyses were conducted using the whole cohort.

After accounting for multiple covariates, the risk for child elevated SBP was higher amongst those whose mothers were in the highest Hcy quartile (Q4) as compared with those in Q2Q3, with an odds ratio (OR) of 1.34 (95\% confidence interval (CI): 1.00,1.81). Children born to obese mothers had higher SBP percentiles and higher odds of elevated SBP (Table 2).

As shown in Figure 2 and Table 3, there was a significant combined effect of maternal Hcy concentration and prepregnancy obesity on offspring SBP percentile and odds of elevated SBP. Children of obese mothers with highest quartile (Q4) Hcy concentrations had a 10.25unit increase $(95 \% \mathrm{CI}, 4.27,16.22)$ in SBP percentile and a 2.22-fold increased risk (95\%CI, $1.35,3.64)$ for elevated SBP compared with children of non-obese mothers with Hcy concentrations in Q2- Q3. There was not a significant interaction between maternal Hcy level and prepregnancy obesity $(\mathrm{P}>0.05)$. Further adjustment for maternal folate and vitamin B12 levels during pregnancy (Table S2), and birth outcome (preterm birth or size at birth, Table S3) in the multivariate logistic models did not alter our findings.

\section{Sensitivity analyses to assess the robustness of the findings}

The associations described above did not differ substantially across strata of race (Black only, Table S4), or child age (6-9 years only, Table S5). For the 702 study children with data on postnatal Hcy concentrations, these children showed lower plasma Hcy concentrations than their mothers: median (IQR): 6.04 (5.15-7.41) umol/L VS. 7.55(6.28-9.57) umol/L. There was a weak correlation between the mother's and child's Hcy level (correlation coefficient: 0.15).The effect of maternal Hcy and prepregnancy obesity on offspring SBP percentile and odds of elevated SBP continued after further adjustment for child Hcy status (Table S6).

\section{Discussion}

To the best of our knowledge, this is the first prospective birth cohort study to assess the combined effect of maternal Hcy concentration and maternal prepregnancy BMI on childhood SBP. Our findings support the premise that maternal Hcy status in the 3rd trimester may play a critical role in child SBP amongst children with obese mothers. 
Our data first revealed a U-shaped association: maternal plasma Hcy concentrations in the lowest quartile (Q1) and highest quartile (Q4) were associated with an increased risk for child elevated SBP as compared with those in Q2-Q3. Based on our thorough literature review, this association between low maternal Hcy and risk of child elevated SBP has not been described before and the underlying mechanisms have not be elucidated. Hcy is a sulfur-containing, non-proteinogenic amino acid. Its metabolism involves two pathways: trans-methylation and trans-sulfuration. ${ }^{33} \mathrm{Hcy}$ is the normal intermediate for conversion of methionine into cysteine, and thus for production of glutathione, taurine and sulfate. Individuals with low Hcy have a restricted ability to respond to oxidative stress and toxins. ${ }^{34,35}$ We speculate that this limited capacity for response to oxidative stress due to low Hcy may enhance the effect of maternal oxidative stress during pregnancy, which may impair fetal growth and increase the risk of child elevated SBP.

High Hcy is common worldwide, especially in low folate regions, ${ }^{31}$ and even in the U.S. In the Framingham Study, the largest longitudinal study conducted on risk factors for cardiovascular disease, the prevalence of high Hcy was $29.3 \%$ for the entire cohort. ${ }^{36}$ In women of childbearing age, a UK study showed that the prevalence of hyperhomocysteinemia was $21.2 \% .{ }^{37}$ Numerous studies have demonstrated the association between elevated Hcy during pregnancy and pregnancy complications ${ }^{38}$, as well as adverse pregnancy outcomes. ${ }^{11,39}$ However, there is a very limited literature regarding the role of maternal Hcy in intergenerational cardiometabolic risk factors like BP. A study of six yearold children found that maternal $1^{\text {st }}$ trimester Hcy concentrations were not associated with childhood BP. ${ }^{40}$ Our study found that elevated maternal Hcy concentrations in the 3rd trimester may increase the harmful effects of maternal prepregnancy obesity on child SBP.

The mechanisms underlying the influence of maternal Hcy on child SBP amongst obese mothers are not clear, but prior research offers some possibilities. One potential mechanism is that maternal prepregnancy obesity can lead to placental inflammation, systemic inflammation and oxidative stress, as demonstrated in two previous studies. ${ }^{41,42}$ Moreover, total oxidation status increases Hcy levels, and is known to generate reactive oxygen species such as $\mathrm{H}_{2} \mathrm{O}_{2}$. Accordingly, it has been proposed that elevated Hcy concentration causes endothelial dysfunction; thus, women who have hyperhomocysteinemia may be likely to experience endothelial dysfunction in the placental vasculature, ${ }^{13}, 43$ which may also have an effect on offspring cardiometabolic health via intrauterine mechanisms. ${ }^{44}$

Elevated Hcy is known to be a modifiable risk factor due to the involvement of vitamin B12 and folate in the transmethylation to methionine. Most times, the use of B12 supplements can return Hcy to an appropriate levels, and may also foster a reduction in related adverse outcomes. However, one study demonstrated increased Hcy levels in mothers of children with neural tube defects even with high circulating folate concentrations, which suggests that there is a direct adverse effect of high Hcy on the developing fetus. ${ }^{45}$ In our study, maternal Hcy had an effect on child SBP independent of maternal folate and vitamin B12 status. These findings support the premise that an elevated Hcy concentration is singularly harmful, for example, via effects on the fetal vascular system. Further studies are needed to elucidate the underlying mechanisms. 
Our study had several limitations. We used maternal plasma Hcy concentrations taken at 2-3 days post-delivery, which is at best a proxy of plasma Hcy during the 3rd trimester of pregnancy. It would be ideal to evaluate maternal Hcy in each trimester given that it changes during pregnancy. Unfortunately, we do not have such data. The question about the role of Hcy in each trimester of pregnancy remains to be determined. While the cardiovascular system develops most rapidly during the 1st trimester, an increase in maternal Hcy concentration and the effect of maternal Hcy on pregnancy complications (hypertensivedisorder) and adverse pregnancy outcomes all mostly occur in the 3rd trimester. ${ }^{11}$ In addition, maternal cardiometabolic risk factors (obesity, hypertensive-disorder, diabetes), poor birth outcome and maternal nutrition in the $3^{\text {rd }}$ trimester are associated with cardiovascular health in childhood. ${ }^{32}$ Although we did not measure fetal Hcy concentrations, a previous study has suggested that the maternal Hcy level is the primary predictor of blood Hcy in the developing fetus. ${ }^{46}$ In our study, we based maternal prepregnancy BMI on selfreported height and weight, thus it may have been subject to reporting bias that led to a misclassification for overweight and obesity. ${ }^{47}$ To assess this possibility, in a subset of the study population ( $\mathrm{N}=672$ ), we compared self-reported BMIs with BMIs extracted from the medical records and found high concordance $(r=0.89, \mathrm{p}<0.001)$. Similarly, maternal plasma Hcy levels are objective measures and their joint association with maternal prepregnancy $\mathrm{BMI}$ on child BP is intriguing and merits additional investigation. Gestational weight gain (GWG) is also a major determinant of offspring health outcomes. However, we have limited data on GWG which resulted in a small sample size for the models with SBP percentiles and elevated SBP and a lack of statistical power. In addition, for a number of reasons the exclusion of 598 children may have resulted in selection bias, although the demographic characteristics of the excluded children were comparable to those of the included children. Another limitation of our study is that it was observational and as such we could not rule out the possibility of residual confounding by unmeasured covariates or confounding, such as other vitamins (methionine, B6, B2, choline and betaine) involved in the DNA methylation cycle, which could have influenced maternal levels of Hcy and childhood SBP.

\section{Perspectives}

In our investigation of this US urban low-income prospective birth cohort, we found that maternal 3rd trimester elevated Hcy and prepregnancy obesity can jointly increase the risk of elevated SBP in children by more than two-fold. Further studies will be needed to replicate these findings and reveal the underlying mechanisms. If further confirmed, screening for Hcy levels in obese mothers may help to identify high risk groups and inform new intervention strategies in order to mitigate intergenerational cardiometabolic risk.

\section{Supplementary Material}

Refer to Web version on PubMed Central for supplementary material.

\section{Acknowledgments}

We wish to thank all of the study participants and the Boston Medical Center Labor and Delivery Nursing Staff for their support and help with the study. We thank T.R. Bartell (Ann \& Robert H. Lurie Children's Hospital of Chicago, Stanley Manne Children's Research Institute, Chicago, USA) for English editing. 
Sources of Funding: The Boston Birth Cohort (the parent study) was supported in part by the March of Dimes PERI grants (20-FY02-56, \#21-FY07-605), and the National Institutes of Health (NIH) grants (R21ES011666, 2R01HD041702, R21HD066471). The follow-up study is supported in part by the NIH grants (U01AI090727, R21AI079872, R01HD086013); and the Maternal and Child Health Bureau (R40MC27443). Dr. Hongjian Wang is supported by a Chinese Scholarships Council scholarship, grants from the National Natural Science Foundation of China (81300156) and PUMC Youth Fund/the Fundamental Research Funds for the Central Universities (3332015103).

\section{References}

1. Jacques PF, Selhub J, Bostom AG, Wilson PW, Rosenberg IH. The effect of folic acid fortification on plasma folate and total homocysteine concentrations. N Engl J Med. 1999; 340:1449-1454. [PubMed: 10320382]

2. Stamler JS, Osborne JA, Jaraki O, Rabbani LE, Mullins M, Singel D, et al. Adverse vascular effects of homocysteine are modulated by endothelium-derived relaxing factor and related oxides of nitrogen. J Clin Invest. 1993; 91:308-318. [PubMed: 8380812]

3. Verhoef P, Stampfer MJ, Buring JE, Gaziano JM, Allen RH, Stabler SP, et al. Homocysteine metabolism and risk of myocardial infarction: Relation with vitamins b6, b12, and folate. Am J Epidemiol. 1996; 143:845-859. [PubMed: 8610698]

4. Castro R, Rivera I, Blom HJ, Jakobs C, Tavares de Almeida I. Homocysteine metabolism, hyperhomocysteinaemia and vascular disease: An overview. J Inherit Metab Dis. 2006; 29:3-20. [PubMed: 16601863]

5. McCully KS. Homocysteine and the pathogenesis of atherosclerosis. Expert Rev Clin Pharmacol. 2015; 8:211-219. [PubMed: 25653125]

6. Shoamanesh A, Preis SR, Beiser AS, Kase CS, Wolf PA, Vasan RS, et al. Circulating biomarkers and incident ischemic stroke in the framingham offspring study. Neurology. 2016

7. Kahleova R, Palyzova D, Zvara K, Zvarova J, Hrach K, Novakova I, et al. Essential hypertension in adolescents: Association with insulin resistance and with metabolism of homocysteine and vitamins. Am J Hypertens. 2002; 15:857-864. [PubMed: 12372672]

8. van Guldener C, Nanayakkara PW, Stehouwer CD. Homocysteine and blood pressure. Curr Hypertens Rep. 2003; 5:26-31. [PubMed: 12530932]

9. Lim U, Cassano PA. Homocysteine and blood pressure in the third national health and nutrition examination survey, 1988-1994. Am J Epidemiol. 2002; 156:1105-1113. [PubMed: 12480655]

10. Vollset SE, Refsum H, Irgens LM, Emblem BM, Tverdal A, Gjessing HK, et al. Plasma total homocysteine, pregnancy complications, and adverse pregnancy outcomes: The hordaland homocysteine study. Am J Clin Nutr. 2000; 71:962-968. [PubMed: 10731504]

11. D'Anna R, Baviera G, Corrado F, Ientile R, Granese D, Stella NC. Plasma homocysteine in early and late pregnancies complicated with preeclampsia and isolated intrauterine growth restriction. Acta Obstet Gynecol Scand. 2004; 83:155-158. [PubMed: 14756732]

12. Steegers-Theunissen RP, Van Iersel CA, Peer PG, Nelen WL, Steegers EA. Hyperhomocysteinemia, pregnancy complications, and the timing of investigation. Obstet Gynecol. 2004; 104:336-343. [PubMed: 15292008]

13. Bukhari SA, Rajoka MI, Ibrahim Z, Jalal F, Rana SM, Nagra SA. Oxidative stress elevated DNA damage and homocysteine level in normal pregnant women in a segment of pakistani population. Mol Biol Rep. 2011; 38:2703-2710. [PubMed: 21107731]

14. McNulty B, McNulty H, Marshall B, Ward M, Molloy AM, Scott JM, et al. Impact of continuing folic acid after the first trimester of pregnancy: Findings of a randomized trial of folic acid supplementation in the second and third trimesters. Am J Clin Nutr. 2013; 98:92-98. [PubMed: 23719554]

15. Flegal KM, Carroll MD, Kit BK, Ogden CL. Prevalence of obesity and trends in the distribution of body mass index among us adults, 1999-2010. JAMA. 2012; 307:491-497. [PubMed: 22253363]

16. Gaillard R, Steegers EA, Duijts L, Felix JF, Hofman A, Franco OH, et al. Childhood cardiometabolic outcomes of maternal obesity during pregnancy: The generation $\mathrm{r}$ study. Hypertension. 2014; 63:683-691. [PubMed: 24379180] 
17. Perng W, Gillman MW, Mantzoros CS, Oken E. A prospective study of maternal prenatal weight and offspring cardiometabolic health in midchildhood. Ann Epidemiol. 2014; 24:793-800 e791. [PubMed: 25263237]

18. Nathanielsz PW, Yan J, Green R, Nijland M, Miller JW, Wu G, et al. Maternal obesity disrupts the methionine cycle in baboon pregnancy. Physiol Rep. 2015; 3

19. Jacques PF, Bostom AG, Wilson PW, Rich S, Rosenberg IH, Selhub J. Determinants of plasma total homocysteine concentration in the framingham offspring cohort. Am J Clin Nutr. 2001; 73:613-621. [PubMed: 11237940]

20. Wang G, Divall S, Radovick S, Paige D, Ning Y, Chen Z, et al. Preterm birth and random plasma insulin levels at birth and in early childhood. JAMA. 2014; 311:587-596. [PubMed: 24519298]

21. Defining adult overweight and obesity. Centers for disease control and prevention. <https:// www.cdc.gov/obesity/adult/defining.Html>

22. Wang X, Zuckerman B, Pearson C, Kaufman G, Chen C, Wang G, et al. Maternal cigarette smoking, metabolic gene polymorphism, and infant birth weight. Jama. 2002; 287:195-202. [PubMed: 11779261]

23. Yu Y, Zhang S, Wang G, Hong X, Mallow EB, Walker SO, et al. The combined association of psychosocial stress and chronic hypertension with preeclampsia. Am J Obstet Gynecol. 2013; 209:438 e431-438 e412. [PubMed: 23850528]

24. Report of the national high blood pressure education program working group on high blood pressure in pregnancy. Am J Obstet Gynecol. 2000; 183:S1-S22.

25. Wang L, Wang X, Laird N, Zuckerman B, Stubblefield P, Xu X. Polymorphism in maternal lrp8 gene is associated with fetal growth. Am J Hum Genet. 2006; 78:770-777. [PubMed: 16642433]

26. National center for health statistics. Cdc growth charts, united states. 2000. http://www.cdc.gov/ growthcharts/

27. Sundstrom J, Neovius M, Tynelius P, Rasmussen F. Association of blood pressure in late adolescence with subsequent mortality: Cohort study of swedish male conscripts. BMJ. 2011; 342:d643. [PubMed: 21343202]

28. Li Z, Snieder H, Harshfield GA, Treiber FA, Wang X. A 15-year longitudinal study on ambulatory blood pressure tracking from childhood to early adulthood. Hypertens Res. 2009; 32:404-410. [PubMed: 19325561]

29. National High Blood Pressure Education Program Working Group on High Blood Pressure in C, Adolescents. The fourth report on the diagnosis, evaluation, and treatment of high blood pressure in children and adolescents. Pediatrics. 2004; 114:555-576. [PubMed: 15286277]

30. Steinberger J, Daniels SR, Eckel RH, Hayman L, Lustig RH, McCrindle B, et al. Progress and challenges in metabolic syndrome in children and adolescents: A scientific statement from the american heart association atherosclerosis, hypertension, and obesity in the young committee of the council on cardiovascular disease in the young; council on cardiovascular nursing; and council on nutrition, physical activity, and metabolism. Circulation. 2009; 119:628-647. [PubMed: 19139390]

31. Huo Y, Li J, Qin X, Huang Y, Wang X, Gottesman RF, et al. Efficacy of folic acid therapy in primary prevention of stroke among adults with hypertension in china: The csppt randomized clinical trial. JAMA. 2015; 313:1325-1335. [PubMed: 25771069]

32. Wang G, Hu FB, Mistry KB, Zhang C, Ren F, Huo Y, et al. Association between maternal prepregnancy body mass index and plasma folate concentrations with child metabolic health. JAMA Pediatr. 2016; 170:e160845. [PubMed: 27295011]

33. Cacciapuoti F. Hyper-homocysteinemia: A novel risk factor or a powerful marker for cardiovascular diseases? Pathogenetic and therapeutical uncertainties. J Thromb Thrombolysis. 2011; 32:82-88. [PubMed: 21234645]

34. Richard S, Lord KF. Significance of low plasma homocysteine. Meametrix Clinical Laboratory, Department of Science and Education. 2006

35. Huang J, Khan S, O'Brien PJ. The glutathione dependence of inorganic sulfate formation from 1- or d-cysteine in isolated rat hepatocytes. Chem Biol Interact. 1998; 110:189-202. [PubMed: 9609386] 
36. Selhub J. The many facets of hyperhomocysteinemia: Studies from the framingham cohorts. J Nutr. 2006; 136:1726S-1730S. [PubMed: 16702347]

37. Sukumar N, Adaikalakoteswari A, Venkataraman H, Maheswaran H, Saravanan P. Vitamin b12 status in women of childbearing age in the uk and its relationship with national nutrient intake guidelines: Results from two national diet and nutrition surveys. BMJ Open. 2016; 6:e011247.

38. Baksu A, Taskin M, Goker N, Baksu B, Uluocak A. Plasma homocysteine in late pregnancies complicated with preeclampsia and in newborns. Am J Perinatol. 2006; 23:31-35. [PubMed: 16450270]

39. Sanchez SE, Zhang C, Rene Malinow M, Ware-Jauregui S, Larrabure G, Williams MA. Plasma folate, vitamin $\mathrm{b}(12)$, and homocyst(e)ine concentrations in preeclamptic and normotensive peruvian women. Am J Epidemiol. 2001; 153:474-480. [PubMed: 11226979]

40. van den Hil LC, Rob Taal H, de Jonge LL, Heppe DH, Steegers EA, Hofman A, et al. Maternal first-trimester dietary intake and childhood blood pressure: The generation $\mathrm{r}$ study. The British journal of nutrition. 2013; 110:1454-1464. [PubMed: 23528150]

41. Huang L, Liu J, Feng L, Chen Y, Zhang J, Wang W. Maternal prepregnancy obesity is associated with higher risk of placental pathological lesions. Placenta. 2014; 35:563-569. [PubMed: 24930988]

42. Malti N, Merzouk H, Merzouk SA, Loukidi B, Karaouzene N, Malti A, et al. Oxidative stress and maternal obesity: Feto-placental unit interaction. Placenta. 2014; 35:411-416. [PubMed: 24698544]

43. Tang L, Mamotte CD, Van Bockxmeer FM, Taylor RR. The effect of homocysteine on DNA synthesis in cultured human vascular smooth muscle. Atherosclerosis. 1998; 136:169-173. [PubMed: 9544744]

44. Roescher AM, Timmer A, Erwich JJ, Bos AF. Placental pathology, perinatal death, neonatal outcome, and neurological development: A systematic review. PLoS One. 2014; 9:e89419. [PubMed: 24586764]

45. van der Put NM, van Straaten HW, Trijbels FJ, Blom HJ. Folate, homocysteine and neural tube defects: An overview. Exp Biol Med (Maywood). 2001; 226:243-270. [PubMed: 11368417]

46. Molloy AM, Mills JL, McPartlin J, Kirke PN, Scott JM, Daly S. Maternal and fetal plasma homocysteine concentrations at birth: The influence of folate, vitamin b12, and the 5,10methylenetetrahydrofolate reductase 677c-->t variant. Am J Obstet Gynecol. 2002; 186:499-503. [PubMed: 11904614]

47. Niedhammer I, Bugel I, Bonenfant S, Goldberg M, Leclerc A. Validity of self-reported weight and height in the french gazel cohort. Int J Obes Relat Metab Disord. 2000; 24:1111-1118. [PubMed: 11033979] 


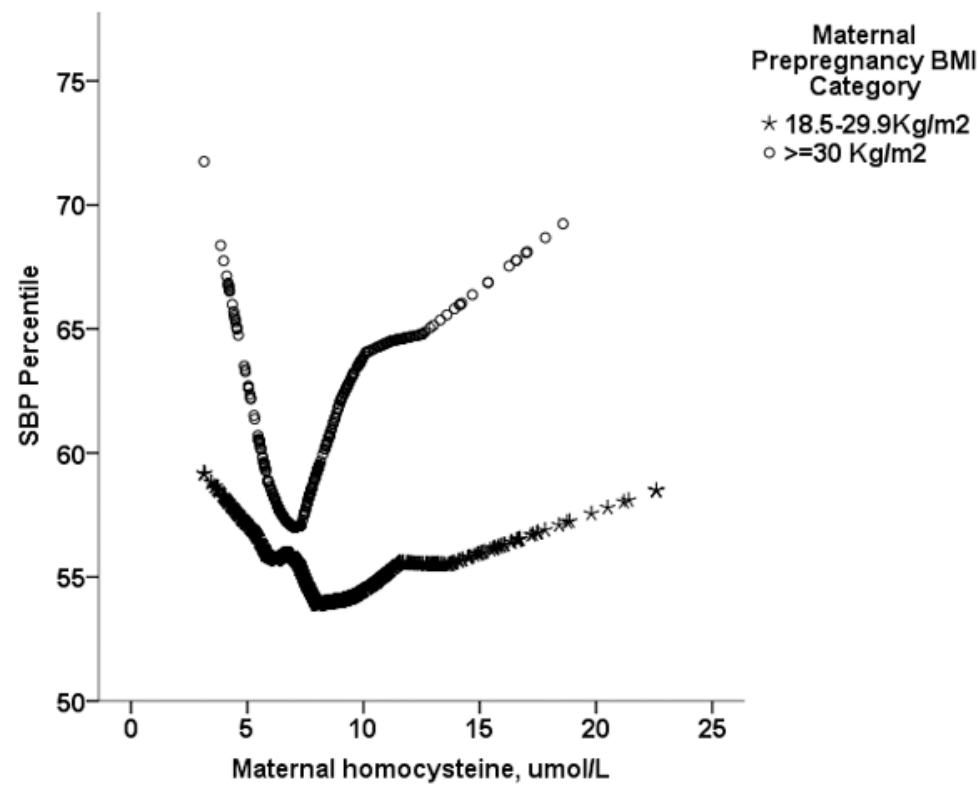

Figure 1.

The association between maternal plasma homocysteine and child SBP percentile stratified by prepregnancy BMI category.

The graph displays the crude association between maternal plasma homocysteine concentration and offspring SBP percentile stratified by maternal prepregnancy BMI category. 

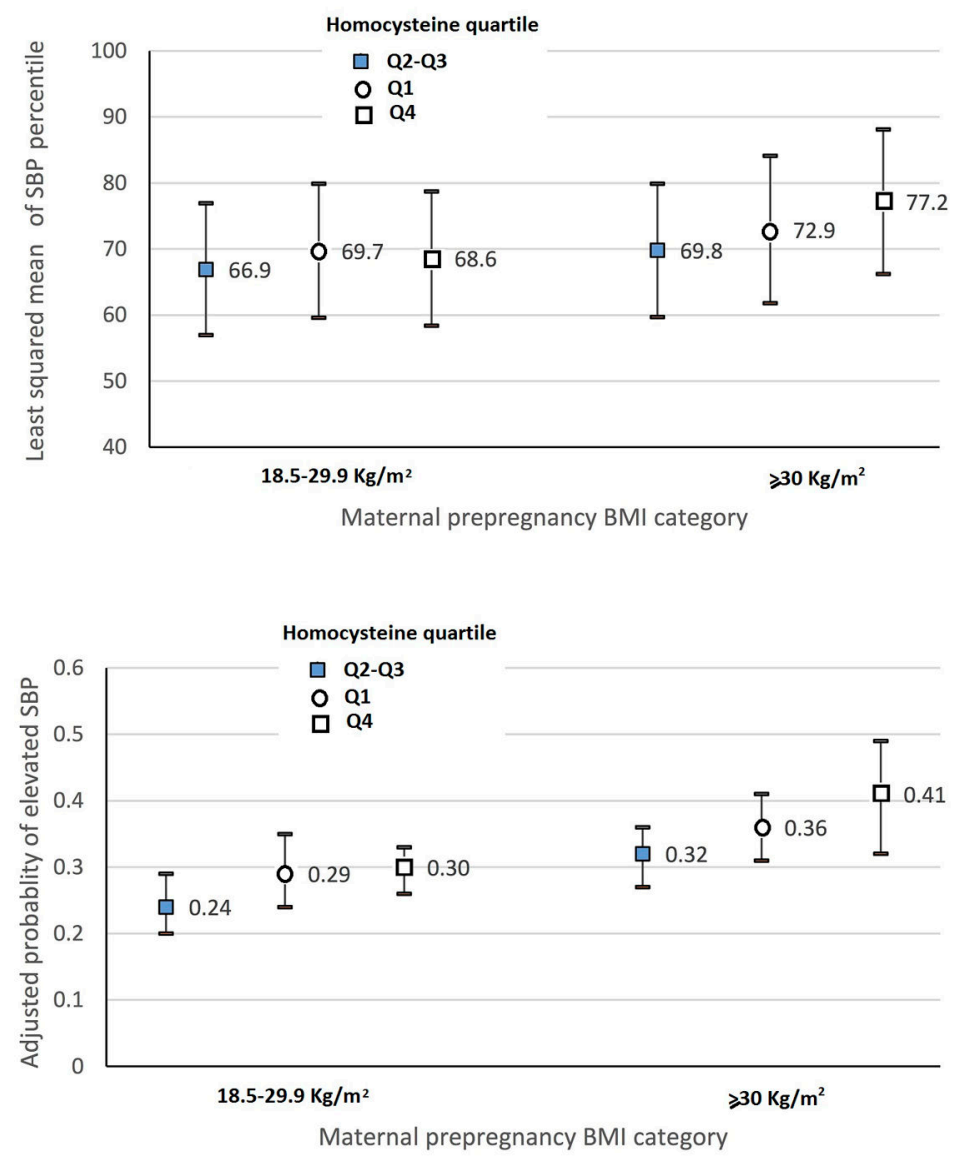

Figure 2.

The combined effect of maternal plasma homocysteine and maternal prepregnancy obesity on SBP percentile and proportion of child elevated SBP (SBP $\geq 75$ th) in the Boston Birth Cohort (BBC)

A, The $y$-axis presents least square means and 95\%CI of child SBP percentiles, as estimated using a generalized linear model with adjustment for maternal age, race/ethnicity, education, smoking, alcohol intake, parity, perceived stress during pregnancy, diabetes and hypertensive disorders. There was no significant interaction between maternal Hcy status and prepregnancy obesity $(\mathrm{P}>.05)$.

$\mathrm{B}$, The $\mathrm{y}$-axis presents adjusted probabilities and 95\%CI of child elevated SBP, as estimated using a logistic regression model with adjustment for the previously mentioned covariates. There was no significant interaction between maternal Hcy status and prepregnancy obesity $(\mathrm{P}>$.05).

Body mass index was calculated as weight in kilograms divided by height in meters squared. The Boston Birth Cohort (BBC) uses a rolling enrollment; the study sample consisted of children enrolled from 1998 to 2012 who have been followed up from birth up to the last visit recorded by electronic medical record. Maternal plasma homocysteine quartile (Q) range: Q1: 3.14 to $6.27 \mathrm{umol} / \mathrm{L}$; Q2: 6.28 to $7.54 \mathrm{umol} / \mathrm{L} ; \mathrm{Q} 3: 7.55$ to $9.57 \mathrm{umol} / \mathrm{L} ; \mathrm{Q} 4: 9.58$ to $22.59 \mathrm{umol} / \mathrm{L}$ 
Table 1

Characteristics of mother-child dyads in the total sample and subgroups stratified by child elevated SBP ( $\mathrm{SBP} \geq 75^{\text {th }}$ percentiles) in the Boston Birth Cohort (BBC)

\begin{tabular}{|c|c|c|c|c|}
\hline Variables & Total Sample & Children without elevated SBP & Children with elevated SBP & P-value \\
\hline No. & 1279 & 910 & 369 & \\
\hline \multicolumn{5}{|l|}{ Maternal Characteristics } \\
\hline Maternal age, years & $28.8(6.6)$ & $28.8(6.5)$ & $28.8(6.9)$ & 0.9048 \\
\hline Race, No. (\%) & & & & 0.6894 \\
\hline Black & $864(67.6)$ & $613(67.4)$ & $251(68.0)$ & \\
\hline Hispanic & $247(19.3)$ & $173(19.0)$ & $74(20.1)$ & \\
\hline Other & $168(13.1)$ & 124(13.6) & $44(11.9)$ & \\
\hline Education, No. (\%) & & & & 0.7221 \\
\hline High School and lower & $831(65.0)$ & $594(65.3)$ & $237(64.2)$ & \\
\hline College and higher & $448(35.0)$ & $316(34.7)$ & $132(35.8)$ & \\
\hline Parity, No. (\%) & & & & 0.9984 \\
\hline Nulliparous & $506(39.6)$ & $360(39.6)$ & 146(39.6) & \\
\hline Multiparous & $773(60.4)$ & $550(60.4)$ & $223(60.4)$ & \\
\hline Smoking, No. (\%) & & & & 0.0357 \\
\hline Never & $1059(82.8)$ & $769(84.5)$ & 290(78.6) & \\
\hline Quitter & $99(7.7)$ & $65(7.1)$ & $34(9.2)$ & \\
\hline Continuous & $121(9.5)$ & $76(8.4)$ & $45(12.2)$ & \\
\hline Alcohol intake, No. (\%) & $100(7.8)$ & $71(7.8)$ & $29(7.9)$ & 0.9726 \\
\hline \multicolumn{2}{|c|}{ Perceived stress during pregnancy, No. (\%) } & & & 0.5566 \\
\hline Low & 1044(81.6) & $736(81.0)$ & $309(83.7)$ & \\
\hline Severe & $235(18.4)$ & $175(19.2)$ & $60(16.3)$ & \\
\hline \multicolumn{2}{|c|}{ Pre-or gestational hypertension, No. (\%) } & & & 0.0308 \\
\hline No & $1093(85.5)$ & $790(86.8)$ & $303(82.1)$ & \\
\hline Yes & $186(14.5)$ & $120(13.2)$ & $66(17.9)$ & \\
\hline \multicolumn{2}{|c|}{ Pre-or gestational diabetes, No. (\%) } & & & 0.3616 \\
\hline No & $1139(89.0)$ & $817(89.8)$ & $322(87.3)$ & \\
\hline Yes & $140(11.0)$ & $93(10.2)$ & $47(12.7)$ & \\
\hline Prepregnancy BMI, $\mathrm{Kg} / \mathrm{m}^{2}$ & $27.2(6.5)$ & $26.8(6.1)$ & $28.2(7.4)$ & 0.0018 \\
\hline \multicolumn{2}{|c|}{ Prepregnancy BMI category, No. (\%) } & & & 0.0039 \\
\hline $18.5-24.9 \mathrm{Kg} / \mathrm{m}^{2}$ & $568(44.4)$ & $414(45.5)$ & 154(41.7) & \\
\hline $25-29.9 \mathrm{~kg} / \mathrm{m}^{2}$ & $389(30.4)$ & 290(31.9) & $99(26.8)$ & \\
\hline $230 \mathrm{~kg} / \mathrm{m}^{2}$ & $322(25.2)$ & $206(22.4)$ & $116(31.4)$ & \\
\hline \multicolumn{5}{|l|}{ Child characteristics } \\
\hline Boy, No. (\%) & $626(48.9)$ & $469(51.5)$ & $157(42.6)$ & 0.0036 \\
\hline Age, year & $6.7(2.1)$ & $6.7(2.1)$ & $6.7(2.1)$ & 0.5256 \\
\hline Birthweight(g) & $2985.5(798.3)$ & $3047.4(751.1)$ & 2833.1(886.9) & $<0.0001$ \\
\hline Gestational age(week) & $37.9(3.4)$ & $38.2(3.0)$ & $37.2(3.9)$ & $<0.0001$ \\
\hline Preterm birth, No. (\%) & $297(23.2)$ & $187(20.6)$ & $110(29.8)$ & 0.0004 \\
\hline
\end{tabular}




\begin{tabular}{lcccc}
\hline Variables & Total Sample & Children without elevated SBP & Children with elevated SBP & P-value \\
\hline \multicolumn{1}{l}{ Birthweight for gestational age, No. $(\%)$} & & & 0.5130 \\
SGA & $151(11.8)$ & $103(11.3)$ & $48(13.0)$ & \\
AGA & $983(76.9)$ & $700(76.9)$ & $283(76.9)$ & \\
LGA & $144(11.3)$ & $107(11.8)$ & $37(10.1)$ & \\
Missing & 1 & 0 & 1 & \\
Weight, Kg & $28.0(11.5)$ & $27.4(10.5)$ & $29.5(13.6)$ & 0.0068 \\
BMI, $\mathrm{Kg} / \mathrm{m}^{2}$ & $18.3(4.0)$ & $17.9(3.6)$ & $19.2(4.8)$ & $<0.0001$ \\
\hline
\end{tabular}

BMI: body mass index; SGA: small for gestational age; LGA: large for gestational age; AGA: appropriate for gestational age. Data are shown as mean (standard deviation, SD) or No (\%). P value is for test of significance (either t-test or chi-squared test) between normal childhood blood pressure and elevated blood pressure group. 

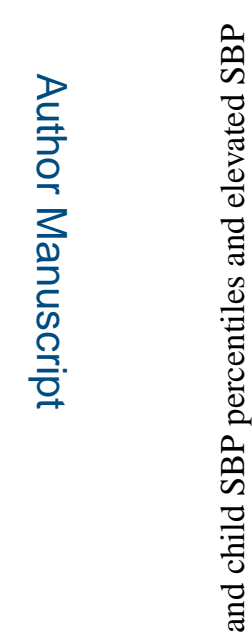

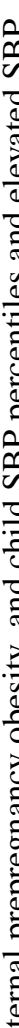
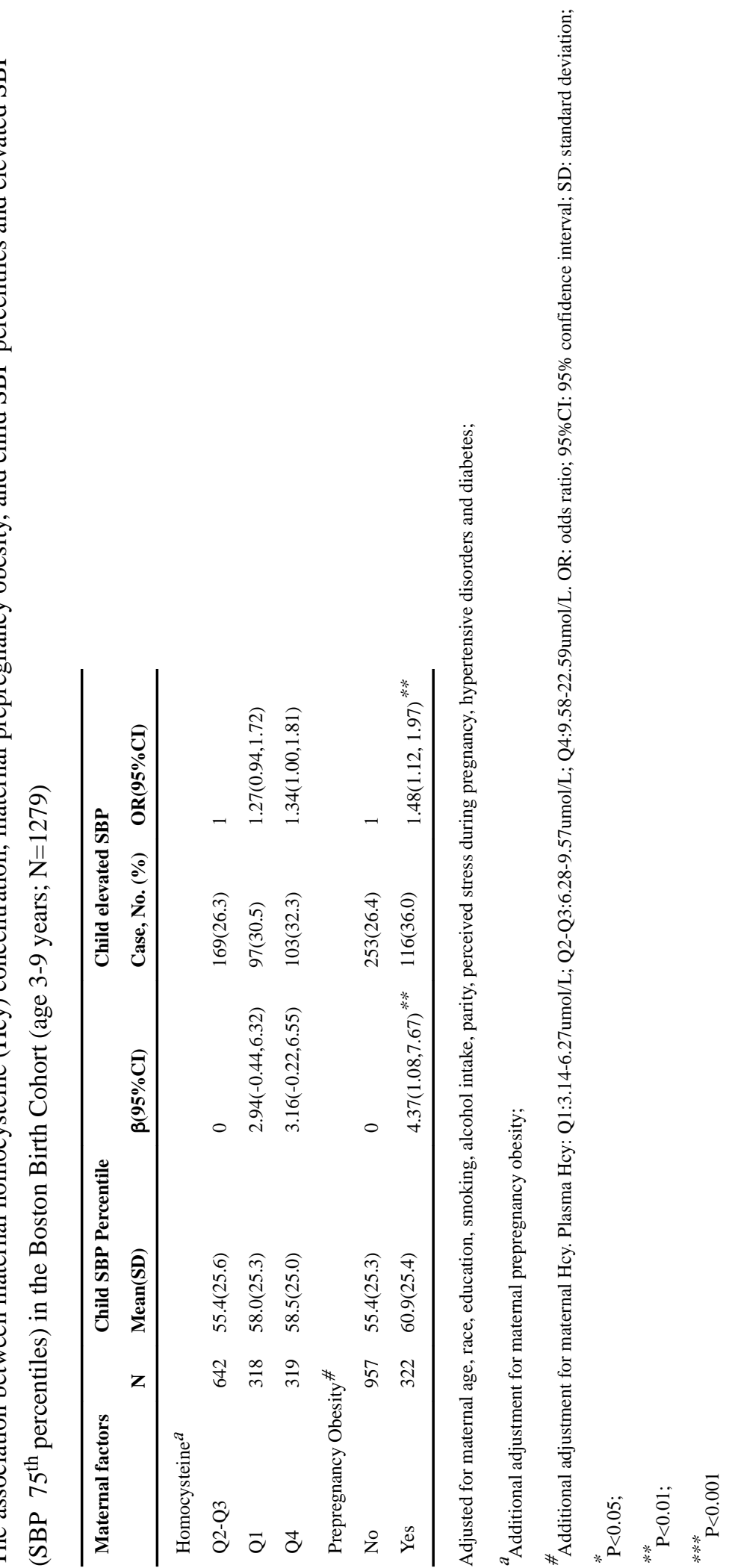

西

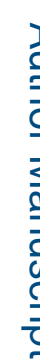

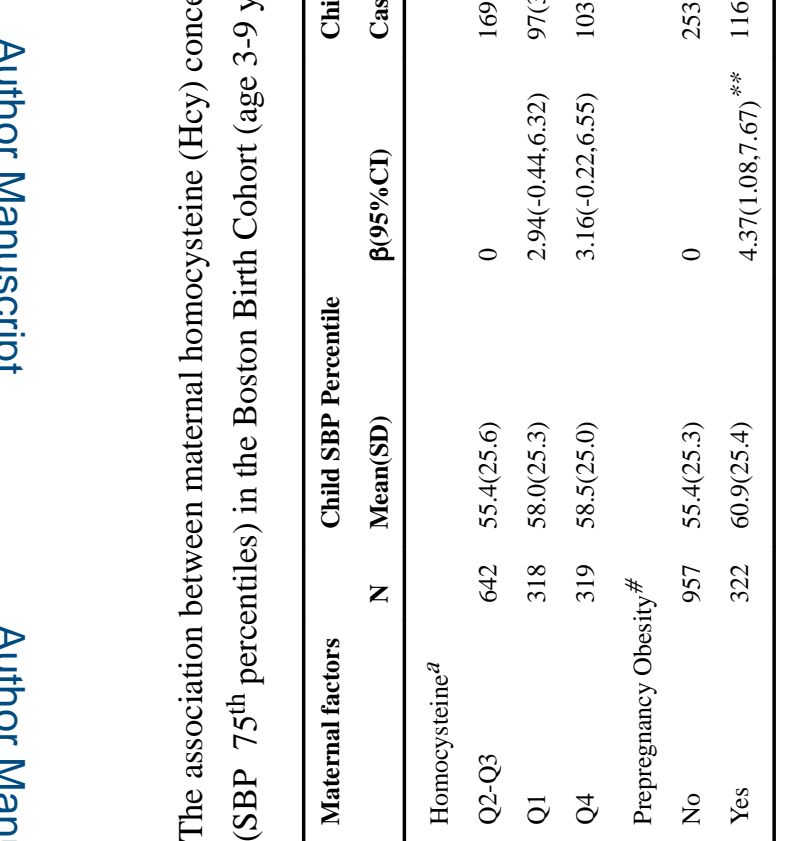$$
\text { 政 }
$$

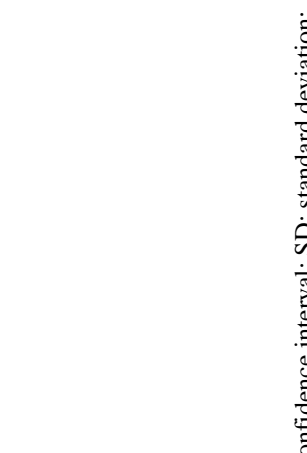




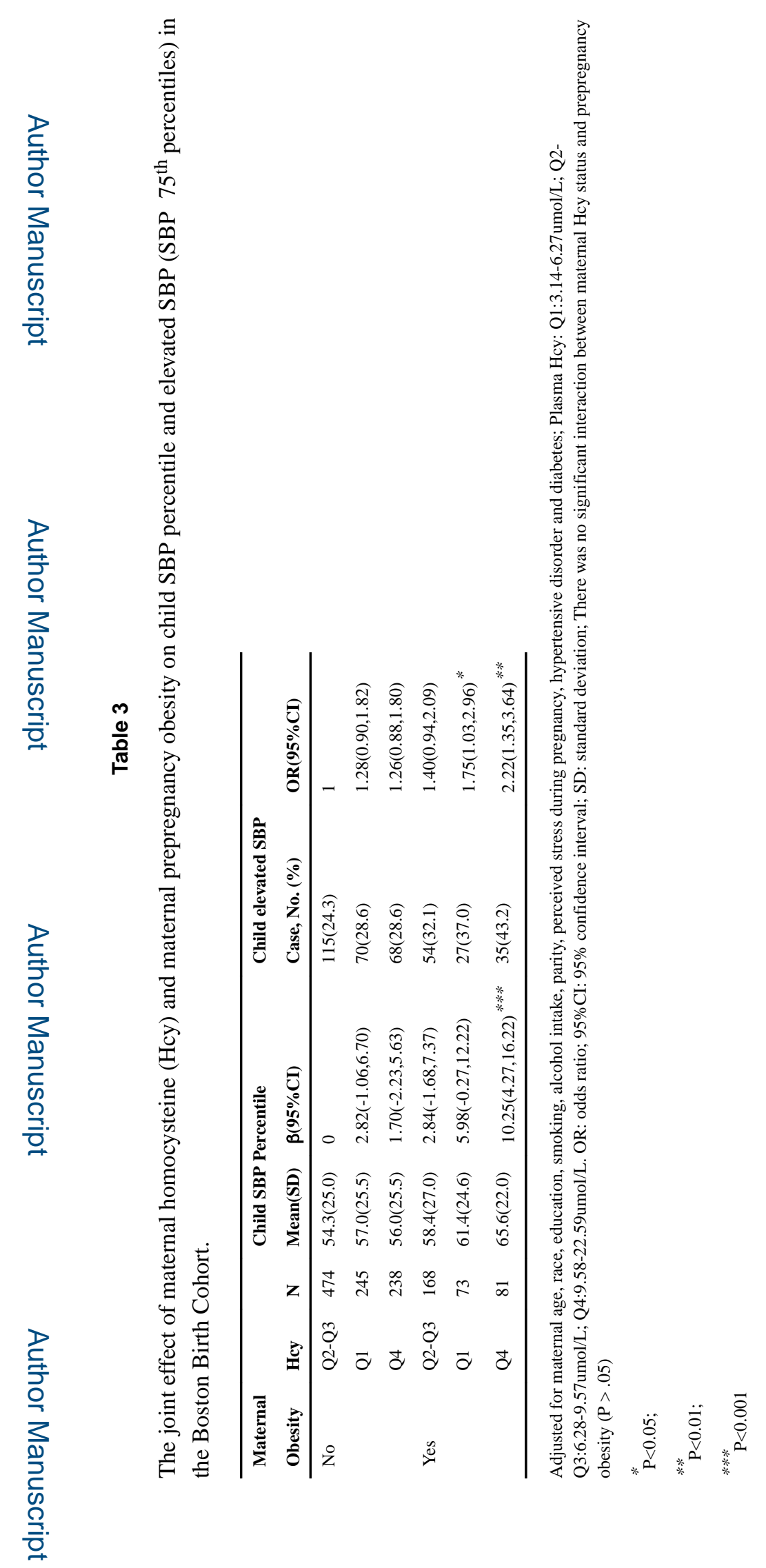

Int J Obes (Lond). Author manuscript; available in PMC 2017 November 03. 